\title{
New capacitive coupled superharmonic quadrature LC-VCO
}

\author{
Emad Ebrahimi ${ }^{\mathrm{a})}$ and Sasan Naseh ${ }^{\mathrm{b})}$ \\ Department of Electrical Engineering, Ferdowsi University of Mashhad \\ Mashhad, Iran \\ a)ebrahimi_em@yahoo.com \\ b)naseh@um.ac.ir
}

Abstract: A study of some reported LC quadrature voltagecontrolled oscillators (LC-QVCO) is performed in which it is shown that robustness of the quadrature oscillation varies depending on the coupling configuration. Next, a new superharmonic LC-QVCO is proposed in which the common source node in either of two identical crossconnected LC-VCOs is coupled via a capacitor to the node common between the two varactors in the LC tank of the other LC-VCO. In the proposed coupling configuration there exists a closed loop through which the second harmonic signals circulate. A qualitative argument is presented to justify the robustness of the quadrature nature of the proposed QVCO by applying the Barkhausen phase criterion to the second harmonic signals in the loop. Since the coupling devices are only two capacitors, no extra noise sources and power consumption are added to the core VCOs. A Monte Carlo simulation showed that the phase error of the proposed QVCO is no more than $1^{\circ}$. Also, generalizing this method to several numbers of VCOs in a loop, multiphase signals can be generated. The proposed circuits were designed using a 0.18- $\mu \mathrm{m} R F$ CMOS technology and simulation results are presented.

Keywords: LC quadrature voltage-controlled oscillators (LC-QVCO), low-phase-noise, multiphase, quadrature robustness, superharmonic QVCO

Classification: Integrated circuits

\section{References}

[1] B. Razavi, RF Microelectronics, Prentice Hall, Upper Saddle River, NJ, 1998.

[2] A. Rofougaran, et al., "A single-chip 900-MHz spread-spectrum wireless transceiver in 1- $\mu \mathrm{m}$ CMOS-Part I: Architecture and transmitted design," IEEE J. Solid-State Circuits, vol. 33, pp. 515-534, April 1998.

[3] P. Tortori, et al., "Quadrature VCOs based on direct second harmonic locking: Theoretical analysis and experimental validation," Int. J. Circuit Theory Appl., DOI:10.1002/cta.612, 2009.

[4] S. Gierkink, S. Levantino, R. Frye, C. Samori, and V. Boccuzzi, "A lowphase-noise 5-GHz CMOS quadrature VCO using superharmonic cou- 
pling," IEEE J. Solid-State Circuits, vol. 38, no. 7, pp. 1148-1154, July 2003.

[5] T. M. Hancock and G. Rebeiz, "A novel superharmonic coupling topology for quadrature design at $6 \mathrm{GHz}, "$ RFIC, pp. 285-288, 2004.

[6] B. Soltanian and P. Kinget, "A low phase noise quadrature LC VCO using capacitive common-source coupling," ESSCIRC, pp. 436-439, 2006.

[7] S. Naseh, M. Zarre, and M. J. Deen, "A Low-Voltage Low-Noise Superharmonic Quadrature Oscillator," IEEE ICECS, pp. 400-403, 2008.

[8] M. Tiebout, "Low-power low-phase-noise differentially tuned quadrature VCO design in standard CMOS," IEEE J. Solid-State Circuits, vol. 36, pp. 1018-1024, July 2001.

[9] B. R. Jackson and C. E. Saavedra, "A $3 \mathrm{GHz}$ CMOS quadrature oscillator using active superharmonic coupling," 37th European Microwave Conference, pp. 1109-1112, 2007.

[10] O. Casha, I. Grech, and J. Micallef, "Comparative study of gigahertz CMOS LC quadrature voltage-controlled oscillators with relevance to phase noise, " Analog Integrated Circuits and Signal Processing, vol. 52, no. 1-2, pp. 1-14, Aug. 2007.

[11] L. Romano, et al., "Phase noise and accuracy in quadrature oscillators," ISCAS, pp. 161-164, 2004.

\section{Introduction}

Many modern communication systems such as zero-IF receivers, image rejection architectures, clock and data recovery (CDR), and QPSK modulators require oscillating signals with $90^{\circ}$ phase differences (I/Q signals) and therefore, quadrature voltage-controlled oscillators (QVCO) are an indispensable part of these systems. Some circuits and methods used for quadrature signals generation are [1]: poly-phase RC-CR filters, master-slave flip-flops used as frequency divider, ring oscillators, and QVCOs with LC tanks (LC-QVCOs).

LC-QVCOs are usually made of coupling two identical cross-connected LC-VCOs. Depending on their mechanism of operation, LC-QVCOs can be categorized in two main groups: first harmonic QVCOs [2] and superharmonic QVCOs $[3,4,5,6,7,8,9]$. In most of the QVCOs in the former group, oscillation frequency deviates from the LC-tank resonance frequency, leading to phase-noise degradation [11]. In superharmonic QVCOs, however, each QVCO operates at its tank resonance frequency and as such, a $3 \mathrm{~dB}$ phase-noise improvement in comparison with that of the core $\mathrm{VCO}$ is observed [3].

\section{Superharmonic coupling mechanisms and quadrature robustness}

Schematics of three different superharmonics QVCOs are shown in Fig. 1 and the common mode node pairs in these circuits are denoted with $\left(a, a^{\prime}\right),\left(b, b^{\prime}\right)$, and $\left(c, c^{\prime}\right)$. As can be easily verified, presence of a $180^{\circ}$ phase difference between the current and/or voltage signals at the common mode nodes of each of the core oscillators, results in $90^{\circ}$ phase differences in the first harmonic 
components of the output signals $[3,4,5,6,7,8,9]$.

The $180^{\circ}$ phase difference can be provided with a transformer. An inverting transformer, as shown in Fig. 1 (a), enforces $180^{\circ}$ phase difference between the potentials of the common source nodes $\left(V_{a}, V_{a^{\prime}}\right)$ [4]. However, the on-chip transformer occupies large chip area and its limited quality factor can cause degradation of the phase-noise.

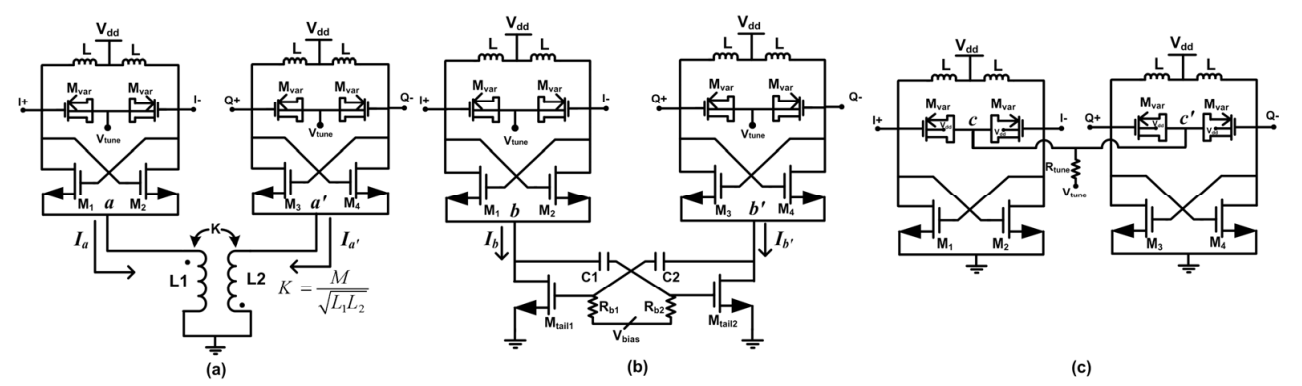

Fig. 1. Schematics of the superharmonic QVCOs (a) and (b): with anti-phase (i.e. $180^{\circ}$ phase difference) coupling $([4,5]),(\mathrm{c})$ with direct coupling $[7]$.

In the QVCO proposed in [5], to save the chip area, the transformer is replaced with a cross-connected differential pair and in this way $180^{\circ}$ phase difference is enforced between the second harmonics of $V_{b}$ and $V_{b^{\prime}}$ (Fig. 1 (b)).

Another way of implementing the superharmonic QVCOs is to couple two identical LC-VCOs by connecting two identical common mode nodes in the core VCOs via passive elements such as capacitor [6], or even via a short circuit [7]. An example is shown in Fig. 1 (c). Further simulations using a wide variety of the circuit parameter values revealed that these circuits $[6,7]$ oscillate in-phase and generate quadrature outputs only for a limited region of circuit elements values, a behavior in contrast to those of $[4,5]$ in which almost always generated quadrature outputs.

In other words, the quadrature nature of the circuits in $[6,7]$ is not as robust as that of the circuits in $[4,5]$. This difference can be summarized and explained as follows. In the circuits in $[6,7]$ it is the nonlinear nature of the injection locking that puts the circuit in the quadrature mode, and the coupling devices play no role in enforcing the $90^{\circ}$ phase differences, while in the circuits in Fig. 1 (a) and (b), besides the injection locking phenomenon, the coupling devices also enforce the $180^{\circ}$ phase differences between the second harmonics.

In this work a new low-phase-noise and robust superharmonic LC-QVCO is proposed.

\section{The proposed superharmonic QVCO}

The proposed QVCO is shown in Fig. 2 (a): two identical LC-VCOs are coupled together via the common source nodes $\left(a, a^{\prime}\right)$ of cross-connected transistors $\left(\mathrm{M}_{1-4}\right)$, and the middle nodes $\left(b, b^{\prime}\right)$ of varactors $\left(\mathrm{M}_{\mathrm{var}}\right)$ in a cross-coupled 
manner, i.e. the second harmonics are injected from node $a$ to node $b^{\prime}$, and from node $b$ to node $a^{\prime}$. This scheme of connection enforces $180^{\circ}$ phase difference between the second harmonics of the two core VCOs and thus, a $90^{\circ}$ phase difference will appear between the fundamental harmonics of the core VCOs at output nodes $\mathrm{I}^{ \pm}$and $\mathrm{Q}^{ \pm}$.

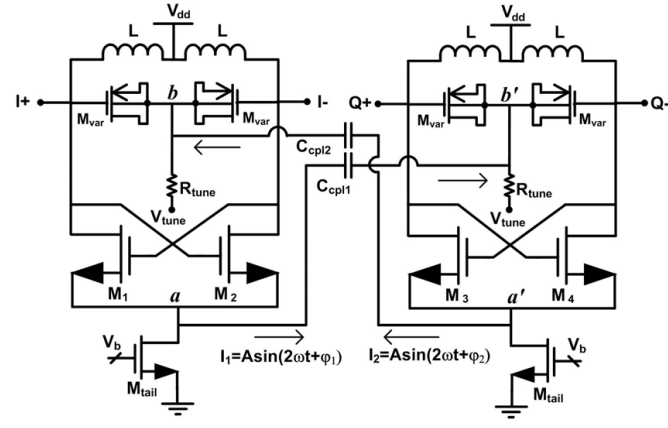

(a)

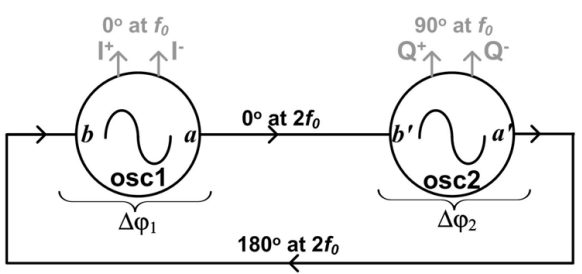

(b)

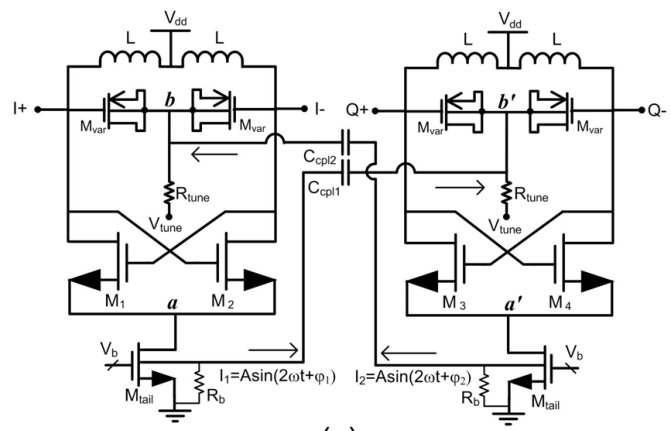

(c)

\begin{tabular}{cc}
\hline \hline Parameter & Value \\
\hline$(\mathrm{W} / \mathrm{L})_{\mathrm{M} 1-4}$ & $8 \mu \mathrm{m} / 0.18 \mu \mathrm{m}$ \\
$(\mathrm{W} / \mathrm{L})_{\mathrm{Mvar}}$ & $280 \mu \mathrm{m} / 0.18 \mu \mathrm{m}$ \\
$\left(\mathrm{W} / \mathrm{L}_{\mathrm{Mtail}}\right.$ & $50 \mu \mathrm{m} / 0.18 \mu \mathrm{m}$ \\
$\mathrm{L}$ & $2 \mathrm{nH}$ \\
$\mathrm{r}_{\mathrm{L}}$ & $2 \Omega$ \\
$\mathrm{R}_{\text {tune }}$ & $1 \mathrm{~K} \Omega$ \\
$\mathrm{C}_{\text {cpl1-2 }}$ & $0.5 \mathrm{pF}$ \\
$\mathrm{V}_{\text {dd }}$ & 1.8 volt \\
$\mathrm{V}_{\text {tune }}$ & 0.8 volt \\
$\mathrm{V}_{\text {bias }}$ & 0.85 volt \\
\hline
\end{tabular}

(d)

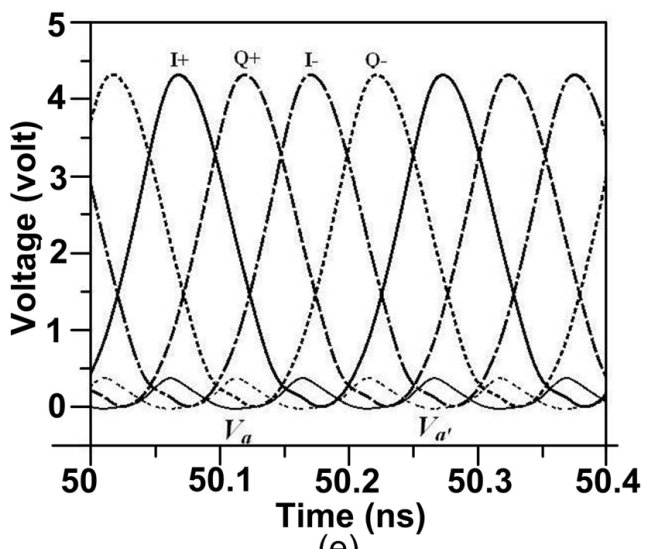

(e)

Fig. 2. (a) Schematic of the proposed superharmonic QVCO, (b) an intuitive model of the proposed QVCO, (c) schematic of another version of the proposed QVCO: using bulk of current sources instead of the source of cross-connected transistors, (d) circuit parameter values of the proposed QVCO used for simulations $\left(\mathrm{r}_{\mathrm{L}}\right.$ is the ohmic loss of the inductors), (e) voltages of the quadrature outputs and the common source nodes of the proposed QVCO in (a). 
The circuit operation can be analyzed from a different point of view as well. The injected second harmonics flow in a closed loop, i.e. $a \rightarrow b^{\prime} \rightarrow$ $a^{\prime} \rightarrow b \rightarrow a$. Depicted in Fig. 2(b), each LC-VCO has been modeled as a black box with two terminals $a$ and $b\left(a^{\prime}\right.$ and $\left.b^{\prime}\right)$ oscillating at frequency $2 f_{0}$. According to the Barkhausen phase criterion, $360^{\circ}$ phase shift will exist around the loop. That is:

$$
\Delta \varphi_{1}+\Delta \varphi_{2}=360^{\circ}
$$

where $\Delta \varphi_{1,2}$ are the phase shifts of the signal through each black box. The two black boxes (LC-VCO) are identical and hence:

$$
\Delta \varphi_{1}=\Delta \varphi_{2}
$$

Equations (1) and (2) simply mean that $\Delta \varphi_{1}=\Delta \varphi_{2}=180^{\circ}$, which, as explained before, leads to a $90^{\circ}$ phase difference between the first harmonics. It can be seen that, while the injection locking phenomenon results in the synchronization of the two core oscillators, presence of the closed loop in this coupling configuration enforces $180^{\circ}$ phase difference between the second harmonics and subsequently results in quadrature output signals. Similar to the QVCOs in $[4,5]$ (in which the utilization of the particular coupling devices resulted in more robust quadrature oscillation compared to those in $[6,7])$, in the proposed QVCO the robustness of the quadrature oscillation is guaranteed due to the presence of the loop; no on-chip transformer or other noisy coupling devices are utilized.

The capacitors $\mathrm{C}_{\mathrm{cpl1}, 2}$ used as the coupling devices in the proposed QVCO introduce no extra noise and power dissipation, and at the same time occupy much less chip area compared to the on-chip transformers [4]. Also, capacitors as coupling devices do not transfer any low frequency noise (e.g. flicker noise) from one core oscillator to the other.

The idea of taking advantage of a loop in the coupling configuration in order to increase the robustness of the quadrature oscillation in superharmonic LC-QVCOs, can be implemented in slightly different forms. One such an example is shown in Fig. 2 (c), in which the substrates of the tail transistors are utilized instead of the common source nodes. The DC biasing of the substrates are provided via the $\mathrm{R}_{b}$ resistors.

\section{Simulation results}

The circuit in Fig. 2 (a) was simulated using the $R F$ models of a commercial 0.18- $\mu \mathrm{m}$ CMOS technology with circuit parameter values shown in Fig. 2 (d). As shown in Fig. 2 (e), there exist $90^{\circ}$ and $180^{\circ}$ phase differences between the outputs $\left(\mathrm{I}^{ \pm}\right.$and $\left.\mathrm{Q}^{ \pm}\right)$of the proposed circuit and between the common source voltages $\left(V_{a}, V_{a^{\prime}}\right)$, respectively. By varying $\mathrm{V}_{\text {tune }}$ from 0 to 1.8 volts, the oscillation frequency changed from 4.75 to $5.25 \mathrm{GHz}$ ( $10 \%$ frequency tuning range). As can be seen in Fig. 3 (a), the phase-noise of the proposed QVCO has almost a flat profile in the whole tuning range. 


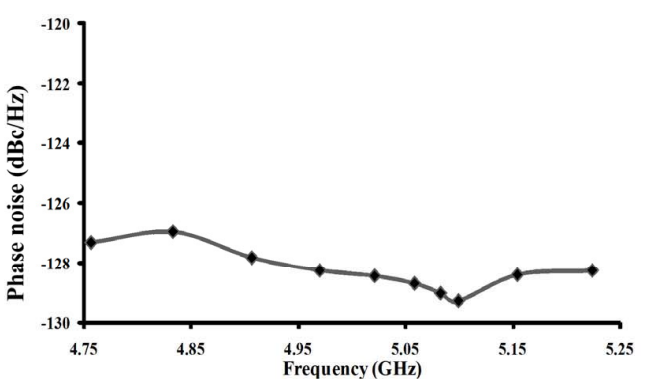

(a)

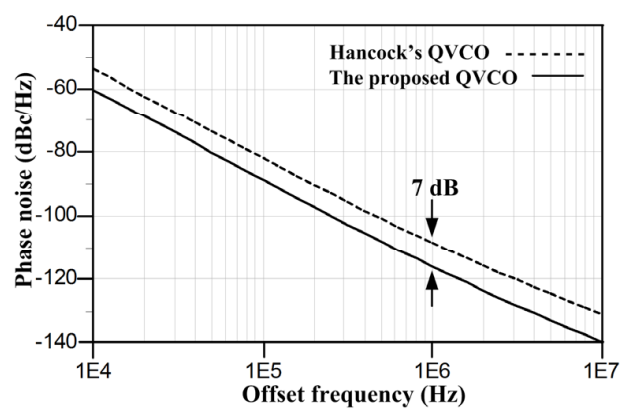

(c)

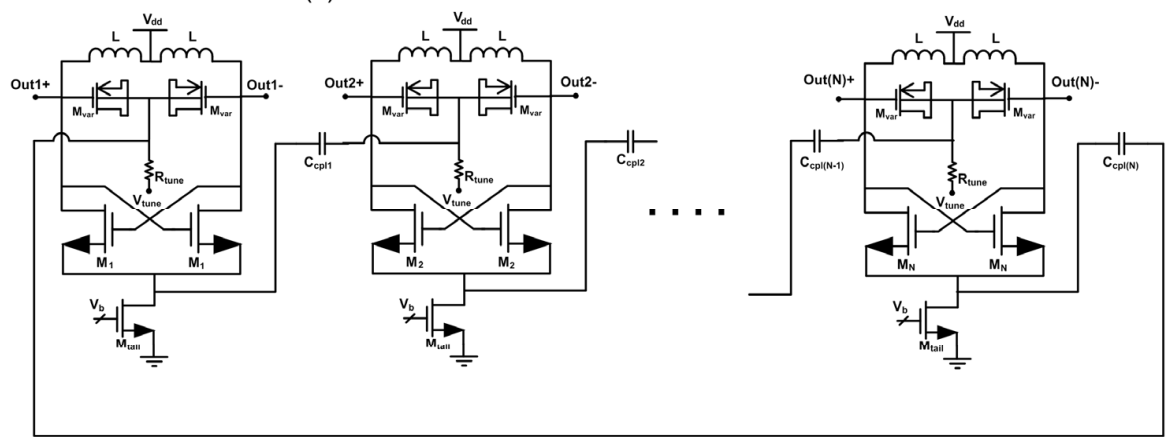

(e)

Fig. 3. (a) Phase noise of the proposed QVCO for tuning range's frequencies (phase noise was measured at $3 \mathrm{MHz}$ frequency offset), (b) Monte Carlo simulation results, (c) phase noise simulation results of the CMOS version of Hancock's QVCO [9] and the proposed QVCO (Fig. 2(a)) at $5 \mathrm{GHz}$ oscillation frequency. (Agilent ADS simulator was used for the simulations.), (d) performance specifications of some previously reported and the proposed QV$\mathrm{COs}$, (e) the proposed multiphase LC-VCO ( $\mathrm{N}$ is an integer number).

To have an estimate of the phase error, a Monte Carlo simulation was performed with $2 \%$ mismatches in the circuit parameters. The results of 30 trials of the Monte Carlo simulation are shown in Fig. 3(b). A maximum of less than $1^{o}$ phase error was observed.

A comparison of the simulated phase-noise of the proposed QVCO (Fig. 2(a)) and that of the CMOS version of Hancock's QVCO [9] is presented in Fig. 3 (c). The proposed QVCO shows a reduction of at least $7 \mathrm{~dB}$ in the phase-noise compared to the QVCO in [9]. This improvement can prob- 
ably be explained by observing that in the QVCOs in [5, 9] each $\mathrm{M}_{\text {tail }}$ acts as a common-source amplifier. Therefore, the noise at the drain of each $\mathrm{M}_{\text {tail }}$ reaches the gate of the other $\mathrm{M}_{\text {tail }}$ and gets amplified, which consequently can significantly degrade the phase-noise of each of the core oscillators. In the proposed QVCO, however, there is no such noise amplification.

In Fig. 3(d) the performance specifications of the proposed QVCOs and several previously reported superharmonic QVCOs are presented. To compare the performance of the different QVCOs, the figure of merit (FOM) suggested in [8] is adopted:

$$
F O M=10 \log \left(S_{S S B}\left(\frac{\Delta f}{f_{\text {osc }}}\right)^{2} P_{Q V C O}\right) .
$$

in which $S_{S S B}$ is the phase-noise at the offset frequency $\Delta f$ in $d B c / H z, f_{\text {osc }}$ is the oscillation frequency and $P_{Q V C O}$ is the total power consumption in milliwatts. (Note: To have a fair comparison, simulation results were used to calculate the FOM for all the circuits, but interestingly, for [4] and [5] the experimental results were better than simulations, so for these two circuits the data presented in the Fig. 2 (d) are based on the reported measurement in those papers.)

Simulations also showed that the proposed QVCOs are able to operate with supply voltages as low as 0.7 volts.

\section{Multiphase signals generation}

The proposed coupling method can be generalized by inserting more than two core oscillators in a loop, as depicted in Fig. 3 (e). Simulations showed that for $\mathrm{N}$ core LC-VCOs in the loop, $2 \mathrm{~N}$ outputs with phases $\mathrm{n} \pi / \mathrm{N}(\mathrm{n}=$ $0,1,2 \ldots 2 \mathrm{~N}-1)$ are generated.

The qualitative analysis of the multiphase VCO made in this way is similar to that of the proposed QVCO (see section 3). Simulations confirmed that it does show the same degree of robustness and phase-noise performance.

To the authors' knowledge, this is the first reported superharmonic multiphase LC-VCO which thereby has the good attributes of the superharmonic coupling $[3,10]$, and at the same time it is robust.

As a typical case, a 3 -stage multiphase $\mathrm{LC}$-VCO was designed with a commercial 0.18- $\mu \mathrm{m} R F$ CMOS process using the proposed coupling method, and simulated with the parameter values in Fig. $2(\mathrm{~d})$. The circuit has a $-130 \mathrm{dBc} / \mathrm{Hz}$ phase-noise at $3 \mathrm{MHz}$ frequency offset from the center frequency of $5 \mathrm{GHz}$, and the total power consumption is less than $15 \mathrm{~mW}$. The FOM is also $-182.5 \mathrm{~dB}$.

\section{Conclusion}

To generate a robust quadrature oscillation a new superharmonic QVCO was proposed in which there exists a loop through which the second harmonic signals circulate. It was shown that the $360^{\circ}$ total phase shift existing in the loop according to the Barkhausen phase criterion, necessitates a $90^{\circ}$ phase 
difference between the output signals, guarantying a robust quadrature signal generation. Generalizing the proposed coupling method to N core LC-VCOs ( $\mathrm{N}$ being an integer number), $2 \mathrm{~N}$ multiphase signals placed in $\pi / \mathrm{N}$ phase interval can be generated. 